\title{
Asociación entre el índice de agua extravascular pulmonar y el balance hídrico en pacientes críticamente enfermos
}

Association between extravascular lung water index and fluid balance in critically ill patients Associação entre água extravascular pulmonar indexada e balanço hídrico em pacientes em estado crítico

Sandybell Sosa Santos, ${ }^{*}$ Luis Antonio Gorordo Delsol, ${ }^{*}$ Marcos A Amezcua Gutiérrez, ${ }^{*}$ Mario Arturo Carrasco Flores, ${ }^{\ddagger}$ José Carlos Gasca Aldama, ${ }^{*}$ Nikolett Iren Medveczky Ordóñez*

\section{RESUMEN}

Introducción: El balance hídrico positivo y el agua extravascular pulmonar medida por termodilución transpulmonar son factores independientes de mortalidad. La inclusión del valor de agua extravascular pulmonar indexada (EVLWi) durante la reanimación inicial impacta en la cantidad de líquido administrado. Aunque no existen antecedentes, buscar una asociación entre ambos parámetros puede llevar a un balance hídrico global negativo guiado por la cantidad de EVLWi. En este estudio retrospectivo se buscó la asociación entre EVLWi y balance hídrico y su impacto en la mortalidad.

Material y métodos: Se realizó un estudio retrospectivo de cohortes con $20 \mathrm{ca}$ sos para establecer la asociación entre agua extravascular pulmonar indexada medida por termodilución transpulmonar y balance hídrico total a las 24, 48 y 72 horas, así como con la mortalidad a 30 días.

Resultados: Un valor de corte $>11 \mathrm{~mL} / \mathrm{kg}$ de EVLWi se asoció con un incremento de la mortalidad; a las 24 horas RR 8.0 (95\% IC 1.2146-52.6944, p = $0.0306)$, a las 48 horas RR 4.3778 (95\% IC 1.1643-15.7177, $p=0.0286)$ y a las 72 horas con RR 3.5000 (95\% IC 0.9497-12.8983, p=0.0598). El valor de corte del balance hídrico fue $\geq 3.5 \mathrm{~L}$, sin asociación con la mortalidad RR $0.1789(95 \%$ IC $0.0125-2.5668, p=0.2054)$ a las 24 horas, RR $0.5000(95 \%$ IC $0.0854-2.9258, p=0.4419)$ a las 48 horas y RR 0.3750 (95\% IC 0.0610-2.3059, $\mathrm{p}=0.2897)$ a las 72 horas. La asociación entre balance hídrico total y EVLWi fue $r^{2}$ Pearson $=0.01269$

Conclusiones: El EVLWi $>11 \mathrm{~mL} / \mathrm{kg}$ se asoció a un incremento en la mortalidad, a diferencia del balance hídrico. No encontramos correlación entre el balance hídrico y el EVLWi.

Palabras clave: Agua extravascular pulmonar, balance hídrico, termodilución, cuidados críticos.

\section{ABSTRACT}

Introduction: Positive fluid balance and extravascular lung water index (EVLWi) quantified by transpulmonary thermodilution have been important independent mortality prognostic factors. Including EVLWi to guide initial fluid reanimation therapy has a high impact in the amount of administered fluid. Although there is not enough evidence, search for an association between EVLWi and fluid balance could lead to a negative fluid balance driven by EVLWi to improve survival rates. In this retrospective study we search for the association between EVLWi, fluid balance and its impact on mortality.

Material and methods: Retrospective, cohort study of 20 cases. We looked for any association between EVLWi by transpulmonary thermodilution and daily fluid balance at 24, 48 and 72 hours and reviewed mortality at 30 days.

Results: An EVLWi cutoff value of $>11 \mathrm{~mL} / \mathrm{kg}$ was associated with a higher mortality; on the first 24 hours with an RR 8.0 (95\% Cl 1.2146-52.6944, $p=$ $0.0306)$, at 48 hours $R R 4.3778(95 \% \mathrm{Cl} 1.1643-15.7177, p=0.0286)$ and at 72 hours RR 3.5000 (95\% Cl 0.9497-12.8983 $p=0.0598$ ). Fluid balance cutoff value was established at $\geq 3.5 \mathrm{~L}$, but we can't find any association with mortality, $R R 0.1789$ (95\% Cl 0.0125-2.5668, $p=0.2054$ ) at 24 hours, $R R 0.5000$ (95\% $\mathrm{Cl} 0.0854-2.9258, p=0.4419)$ at 48 hours y $R R 0.3750$ (95\% Cl 0.0610-2.3059, $p=0.2897$ ) at 72 hours. The correlation between fluid balance and EVLWi was negative, Pearson's $r^{2}=0.01269$.

Conclusions: EVLWi was associated to a higher mortality. We could no demonstrate an association between fluid balance and EVLWi.

Keywords: Extravascular lung water, fluid balance, thermodilution, critical care.

* Hospital Juárez de México OPD. Ciudad de México, México.

‡ Sanatorio Durango. Ciudad de México, México.

Recepción: 30/08/2019. Aceptación: 15/10/2019.

www.medigraphic.com/medicinacritica

\section{RESUMO}

Introdução: O balanço hídrico positivo e a água extravascular pulmonar medida por termodiluição transpulmonar são fatores independentes da mortalidade. A inclusão do valor de água extravascular pulmonar indexada (EVLWi) durante a ressuscitação inicial impacta a quantidade de fluido entregue. Embora não haja precedente, buscar uma associação entre os dois parâmetros pode levar a um balanço hídrico global negativo guiado pela quantidade de EVLWi. Neste estudo retrospectivo, buscou-se a associação entre EVLWi e balanço hídrico e seu impacto na mortalidade.

Material e métodos: Foi realizado um estudo de coorte retrospectivo com 20 casos para estabelecer a associação entre a água pulmonar extravascular indexada medida por termodiluição transpulmonar e balanço hídrico total em 24, 48 e 72 horas, bem como mortalidade em 30 dias.

Resultados: Um valor de corte > $11 \mathrm{~mL} / \mathrm{kg}$ de EVLWi foi associado a um aumento na mortalidade; às 24 horas RR 8.0 (95\% IC 1.2146-52.6944, $p=$ $0.0306), 48$ horas RR $4.3778(95 \%$ IC 1.1643-15.7177, $p=0.0286)$ e 72 horas com RR 3.5000 (95\% IC 0.9497-12.8983 $p=0.0598)$. O valor de corte do balanço hídrico foi $\geq 3.5 \mathrm{~L}$, sem associação com mortalidade $R R 0.1789$ (IC $95 \% 0.0125-2.5668, p=0.2054$ ) em 24 horas, $R R 0.5000$ (IC 95\% 0.0854$2.9258 p=0.4419$ ) em 48 horas e RR 0.3750 (IC 95\% 0.0610-2.3059 $p=$ $0.2897)$ às 72 horas. A associação entre balanço hídrico total e EVLWi foi $r^{2}$ Pearson $=0.01269$.

Conclusões: EVLWi > $11 \mathrm{~mL} / \mathrm{kg}$ foi associado a um aumento da mortalidade, em contraste com o balanço hídrico. Não encontramos correlação entre o balanço hídrico e o EVLWi.

Palavras-chave: Água pulmonar extravascular, balanço hídrico, termodiluição, cuidados intensivos.

\section{INTRODUCCIÓN}

Debido a que la optimización de la volemia es pilar fundamental en la estabilización hemodinámica del paciente en estado crítico, una adecuada reanimación con líquidos es de vital importancia en la Unidad de Cuidados Intensivos (UCI). La terapia hídrica intravenosa salva vidas; sin embargo, en pacientes críticamente enfermos la combinación de una enérgica reanimación hídrica y oliguria resulta en acumulación excesiva de líquido que conlleva a un balance hídrico global positivo. La asociación entre la sobrecarga hídrica y la mortalidad está bien documentada, Acheampong y Vincent y colaboradores ${ }^{1}$ estudiaron un grupo de 173 pacientes cuyos ingresos diarios fueron mayores en los no-sobrevivientes que en los sobrevivientes ( $59 \pm 24$ vs. $48 \pm 23 \mathrm{~mL} / \mathrm{kg}, \mathrm{p}=0.03$ ); sin embargo, los egresos fueron similares. Como resultado la persistencia de un balance hídrico global positivo se asoció a un incremento en la mortalidad.

El agua extravascular pulmonar (EVLW) es la cantidad de agua contenida fuera de la vasculatura pulmonar y corresponde a la suma del fluido intersticial, intrace- 
lular, alveolar y linfático, sin incluir los derrames pleurales. Durante muchos años se han buscado técnicas de medición del EVLW, como primera alternativa clínica la dilución transpulmonar termo-colorante, que puede considerarse como el estándar de oro in vivo para la medición de EVLW, ésta se ha desarrollado y validado frente a la gravimetría en animales ${ }^{2}$ y seres humanos. ${ }^{3}$ La técnica requiere un catéter venoso central insertado en el territorio de la vena cava superior y un catéter arterial de punta termistor colocado en la arteria femoral. La dilución del colorante térmico se realiza inyectando simultáneamente a través del catéter venoso central un indicador de frío (solución salina fría) y un indicador colorimétrico (verde de indocianina). El volumen de distribución del indicador de frío incluye los espacios intravascular y extravascular del compartimiento intratorácico, mientras que el indicador colorimétrico es estrictamente un indicador intravascular. ${ }^{4}$ Por lo tanto, la medición de EVLW se obtiene restando el volumen de distribución de estos dos indicadores. En el pulmón sano, el valor normal del agua extravascular pulmonar indexado al peso corporal (EVLWi) es $<7 \mathrm{~mL} / \mathrm{kg}$, esto resulta del equilibrio en la fuga de líquido y el drenaje linfático. ${ }^{5}$

La sobrecarga hídrica es un factor independiente de mortalidad, reflejado como edema intersticial, derrame pleural y aumento de EVLWi, que toma mayor importancia en los pacientes críticamente enfermos que presentan endoteliopatía, aumento de la permeabilidad de la barrera alveolocapilar y disminución de la eliminación del líquido alveolar que llevan a un incremento en la presión hidrostática pulmonar reflejada como un valor $>$ 2 de índice de permeabilidad vascular pulmonar (IPVP). Debido a esto, toma importancia el valor de EVLWi en la mortalidad. En un estudio realizado por Sakka y colaboradores ${ }^{6}$ en 2002 el EVLWi fue un predictor independiente de supervivencia, se analizaron 373 pacientes, el EVLWi fue significativamente mayor en pacientes no sobrevivientes que en los sobrevivientes (media de 14.3 vs. $10.2 \mathrm{~mL} / \mathrm{kg}, p>0.001$ ), un punto de corte establecido $>6.5 \mathrm{~mL} / \mathrm{kg}$ resultó en una sensibilidad de $69.4 \%$ y especificidad de $50.8 \%$.

Es indudable el valor pronóstico del balance hídrico y del valor de EVLWi en los pacientes críticamente enfermos, ambos como un factor independiente de mortalidad, en la actualidad en los pacientes hipoxémicos la inclusión del EVLWi en la decisión de terapia hídrica tiene un alto impacto en la cantidad de fluido administrado, aunque no existe evidencia clara, suena lógico buscar un balance hídrico global negativo guiado por la cantidad de EVLWi y su comportamiento durante la estancia hospitalaria. En 2014 L. Díaz Rubia y colaboradores realizaron un estudio observacional de 44 pacientes, en el cual se analizó el impacto de una estrategia de balance negativo en la función respiratoria, hemodi- námica y renal. ${ }^{7}$ Se observó que en grupo de pacientes con balance hídrico negativo el EVLW descendió de 14 a $10 \mathrm{~mL} / \mathrm{kg}(p>0.001)$, asimismo se observó mejoría en la $\mathrm{PaO}_{2} / \mathrm{FiO}_{2}$, niveles de PEEP, presión plateau e índice de permeabilidad vascular pulmonar. Los pacientes que lograron un balance hídrico negativo presentaron mejoría en la función respiratoria así como una significativa reducción del EVLWi. Por lo tanto, se puede concluir que en los pacientes críticamente enfermos los balances hídricos negativos disminuyen la presión capilar pulmonar, por lo tanto bajan el valor de EVLWi pudiendo dar lugar a una correlación entre ambos valores, por lo que realizar un estudio entre la asociación del balance hídrico y el valor de EVLWi puede arrojar nuevos patrones de análisis en el manejo de fluidos así como llevar a un protocolo de restricción hídrica basado en valores de EVLWi.

\section{MATERIAL Y MÉTODOS}

Se realizó un análisis retrospectivo de 20 pacientes ingresados a la $\mathrm{UCI}$ con diagnóstico de choque séptico de origen respiratorio y no respiratorio en quienes se colocó monitoreo hemodinámico continuo $\left(\mathrm{EV} 1000^{\circledR} \mathrm{de}\right.$

Tabla 1: Variables demográficas, balance y termodilución transpulmonar.

\begin{tabular}{lccc}
\hline Variable & $\begin{array}{c}\text { No } \\
\text { sobrevivientes } \\
n=9\end{array}$ & $\begin{array}{c}\text { Sobrevivientes } \\
n=11\end{array}$ & $\mathrm{p}$ \\
\hline Edad & 41.56 & 39.18 & 0.3538 \\
Sexo & & & \\
$\quad$ Masculino & 5 & 7 & 0.7140 \\
$\quad$ Femenino & 4 & 4 & \\
IMC & 31.61 & 29.05 & 0.1402 \\
Diagnóstico & & & \\
Pulmonar & 5 & 2 & 0.936 \\
No pulmonar & 4 & 9 & \\
Balance hídrico & & & \\
global (día) & & $3,212.17$ & 0.1238 \\
1 & 991.11 & $1,946.54$ & 0.2947 \\
2 & 735.6 & $1,439.08$ & 0.4663 \\
3 & 1,671 & & \\
EVLW (día) & & 10.03 & 0.0041 \\
1 & 20.70 & 10.55 & 0.0043 \\
2 & 19.27 & 10.38 & 0.0054 \\
3 & 23.99 & & \\
IPVP (día) & & 2.56 & 0.0151 \\
1 & 4.03 & 2.48 & 0.0295 \\
2 & 3.74 & 2.32 & 0.0043 \\
3 & 4.25 & 6 & 0.3470 \\
TRRC & 3 & 5.7 & 0.4662 \\
Días de AMV & 5.63 & 7 & 0.3121 \\
Días de estancia & 5.67 & & \\
en la UCl & & & \\
\hline IMC & & & \\
\hline
\end{tabular}

IMC = índice de masa corporal; EVLW = agua pulmonar extravascular; IPVP = índice de permeabilidad vascular pulmonar; TRRC = terapia de reemplazo renal continua; $\mathrm{AMV}=$ asistencia mecánica ventilatoria; $\mathrm{UCl}=$ Unidad de Cuidados Intensivos. 


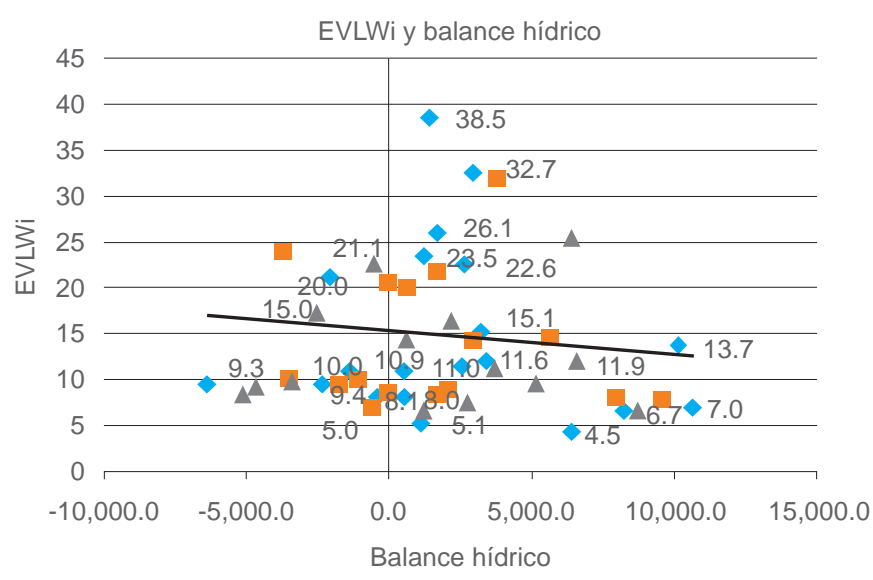

$$
\begin{aligned}
& \text { Día } 1 \longrightarrow \text { Día } 2 \underset{\text { Día } 3}{y=-0.0003 x+15.399} \quad r^{2}=0.0127
\end{aligned}
$$

Figura 1: Dispersión y correlación entre balance hídrico y EVLWi por día.

Edwards Lifesciences ${ }^{\mathrm{TM}}$ ), se realizó cuantificación de variables hemodinámicas estáticas y dinámicas por inyección de medio frío por termodilución transpulmonar (EVLWi y IPVP) a las 24, 48 y 72 horas de su ingreso a la UCl así como cuantificación de balance hídrico global durante los primeros tres días de estancia. Se capturaron las variables en Microsoft Excel ${ }^{\circledR}$ y se utilizó estadística descriptiva para el análisis de las variables demográficas y desenlaces buscados. Se utilizó un análisis $\mathrm{r}^{2}$ de Pearson para establecer la relación entre el EVLWi y el balance hídrico global. El protocolo fue revisado y aprobado por el Comité de Investigación con registro HJM 0582/19-R; el manuscrito se preparó según las recomendaciones de lista de cotejo STROBE. ${ }^{8}$

\section{RESULTADOS}

Se analizaron datos de 20 pacientes, en los cuales la mortalidad a 30 días fue de $45 \%(n=9)$, con un total de siete pacientes con choque séptico pulmonar (SIRA) y 13 pacientes con choque séptico de origen no pulmonar, mortalidad de 71.4 vs. $30.76 \%$. El promedio de valor de EVLWi en el grupo de no sobrevivientes fue de 21.32 vs. 10.32 en el grupo de sobrevivientes, asimismo se presentó mayor valor de IPVP en el grupo de no sobrevivientes, cuatro vs. 2.45. Dentro de las intervenciones realizadas $33.3 \%(n=3)$ de pacientes del grupo de no sobrevivientes recibieron terapia de reemplazo renal continua vs. $54.59 \%(n=6)$ en el grupo de sobrevivientes (Tabla 1). Se realizó una correlación de $\mathrm{r}^{2}$ Pearson para calcular la asociación entre EVLWi y balance hídrico acumulado en el día 1, 2 y 3, con una ${ }^{2}$ de Pearson $=0.01260$, por lo que no existe asociación entre estos dos parámetros (Figura 1).

El comportamiento del agua extravascular pulmonar indexada en los pacientes no sobrevivientes fue mayor de $11 \mathrm{~mL} / \mathrm{kg}$ (media $20.7 \mathrm{~mL} / \mathrm{kg}$ día $1.20 \mathrm{~mL} / \mathrm{kg}$ día 2 y $18.3 \mathrm{~mL} / \mathrm{kg}$ en día 3) y los sobrevivientes presentaron valores de EVLWi menores de $11 \mathrm{~mL} / \mathrm{kg}$ (media 10, 10.6 y $10.4 \mathrm{~mL} / \mathrm{kg}$ a las 24,48 y 72 horas correspondientemente). Se observó un valor máximo de EVLWi de $23.5 \mathrm{~mL} / \mathrm{kg}$ y en los pacientes con choque séptico de origen pulmonar, los valores fueron 15.1-26.1 mL/ $\mathrm{kg}$. Se estableció un valor de corte $>11 \mathrm{~mL} / \mathrm{kg}$ que fue asociado a mayor mortalidad; en las primeras 24 horas con un RR 8.0 (95\% IC 1.2146-52.6944, $p=0.0306$ ), a las 48 horas RR 4.3778 (95\% IC 1.1643-15.7177, $\mathrm{p}=0.0286)$ y a las 72 horas con RR 3.5000 (95\% IC 0.9497-12.8983, $\mathrm{p}=0.0598$ ) (Figura 2).

No se observó una relación entre el valor de balance hídrico global y la mortalidad, se estableció un valor de corte $\geq 3.5 \mathrm{~L}$ y se obtuvieron los siguientes resultados: RR 0.1789 (95\% IC 0.0125-2.5668, $\mathrm{p}=0.2054$ ) en día 1, en el día 2 RR 0.5000 (95\% IC 0.0854-2.9258, $\mathrm{p}=0.4419)$ y RR 0.3750 (95\% IC 0.0610-2.3059, $\mathrm{p}=$ 0.2897 ) el día 3. Los pacientes no sobrevivientes presentaron balances hídricos menores en comparación con los sobrevivientes; sin embargo, no existió una disminución progresiva de éstos durante su estancia en la $\mathrm{UCl}$, además de que presentaban un notable aumento en los valores de IPVP (>2) en comparación con los sobrevivientes (<2) [(día 1: RR 2.6667 95\% IC 0.433916.3904, $p=0.2897$ ), (día 2: RR $0.875095 \%$ IC 0.2836 2.6994, $p=0.8163$ ), (día 3: RR 2.86 95\% IC 0.7794 $-10.5218, p=0.1131$ )] (Figura 3).

\section{DISCUSIÓN}

De acuerdo con los resultados obtenidos en este estudio, no se observa asociación entre el EVLWi y el balance hídrico acumulado en los primeros tres días de estancia en la UCI al obtener una $r^{2}$ de Pearson = 0.01269 , a pesar de que no existen estudios similares en la literatura, el resultado esperado sería encontrar

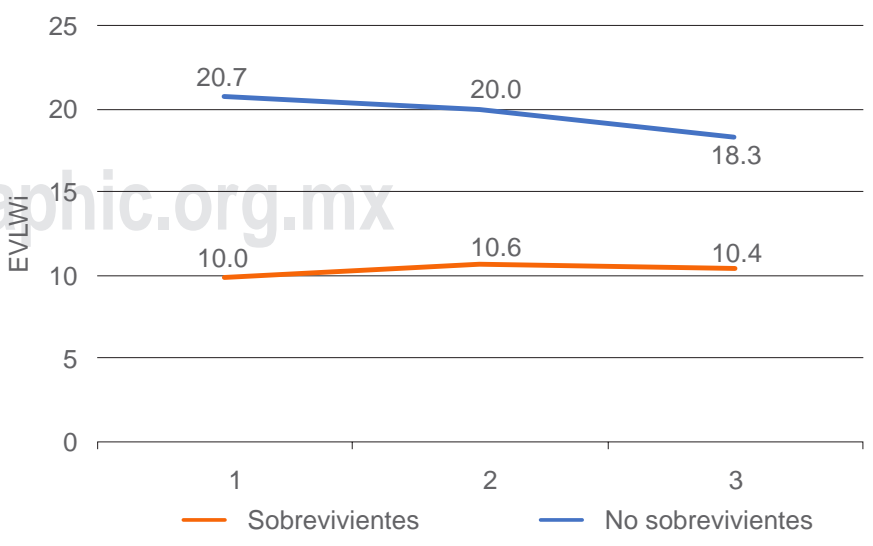

Figura 2: Comportamiento (medias) del EVLWi de los tres primeros días entre sobrevivientes y no sobrevivientes. 


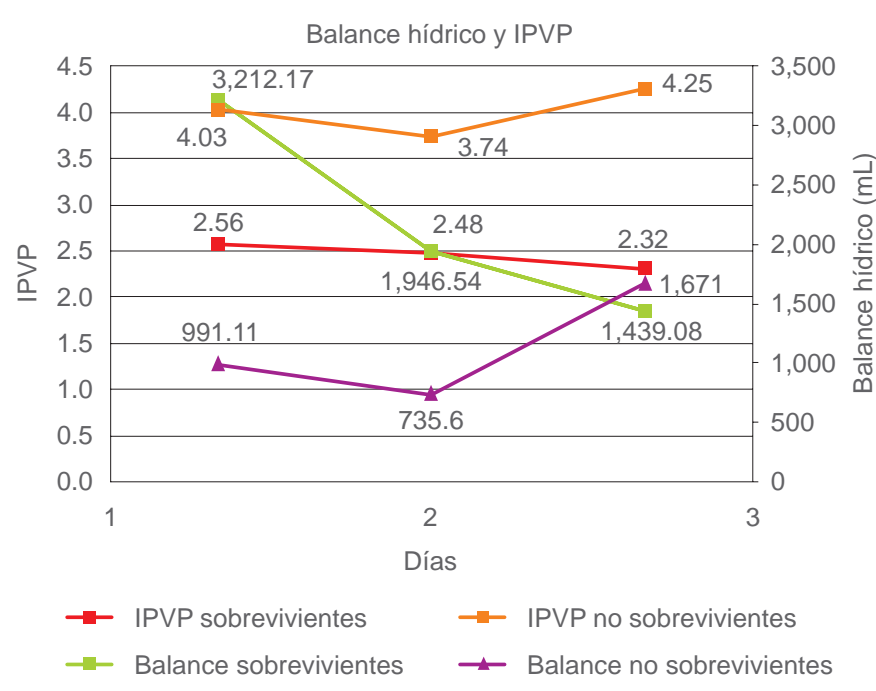

Figura 3: Comportamiento (medias) de IPVP y balance hídrico durante los tres primeros días de estancia de sobrevivientes y no sobrevivientes.

una asociación de los balances hídricos positivos acumulados y el valor positivo de EVLWi; sin embargo, no existe un punto de corte para asociar estas dos variables. L Díaz-Rubia y colaboradores ${ }^{7}$ publicaron en 2014 un estudio observacional de 44 pacientes, en el cual realizaron maniobras para lograr un balance hídrico negativo de acuerdo con el valor de EVLWi. En este estudio se observó una mejoría del índice de $\mathrm{PaO}_{2} /$ $\mathrm{FiO}_{2}$ y en los parámetros ventilatorios, además de una disminución del valor de EVLWi en los pacientes que lograron llevarse a un balance hídrico acumulado negativo; por el contrario, los pacientes que permanecieron con balance hídrico positivo no presentaron cambios en parámetros hemodinámicos o metabólicos, similar a nuestros resultados en este estudio, donde observamos mejoría en la sobrevida en pacientes con balances hídricos positivos que tuvieron tendencia a la negativización, a diferencia de los no sobrevivientes que presentaron balances hídricos persistentemente positivos, sin obtenerse un valor significativo en el riesgo relativo de mortalidad. Estos resultados difieren a lo encontrado por Acheampong y Vicent en $2015,{ }^{1}$ quienes demostraron mayor mortalidad asociada con un balance hídrico positivo persistente con un HR 1.014 (95\% IC 1.0071.022) por cada $\mathrm{mL} / \mathrm{kg}$ de balance hídrico acumulado > $10 \%$ de agua corporal total (ACT); en cambio en nuestro estudio no se encontró dicha asociación; sin embargo, las intervenciones realizadas en los pacientes que sobrevivieron muestran una clara tendencia a la negativización de los balances hídricos, que al final resultaron en balances hídricos similares a los no sobrevivientes. De igual forma se observó que el IPVP fue considerablemente mayor en los pacientes que fallecieron, esto tal vez como consecuencia del estado proinflamatorio y la endoteliopatía inducida por choque, situaciones que condicionan aumento en el índice de fuga capilar y extravasación de líquidos. Por lo tanto, se puede deducir que a pesar de que estos pacientes no fueron sometidos a una acumulación excesiva de líquidos, presentaron mayores valores de EVLWi y por lo tanto, mayor riesgo de mortalidad.

Por otro lado, se observó una clara asociación entre el EVLWi y la mortalidad, los valores de EVLWi en el grupo de sobrevivientes fueron de 10.00-10.55 mL/ $\mathrm{kg}$ vs. no sobrevivientes de $20-24 \mathrm{~mL} / \mathrm{kg}, \mathrm{p}=0.0041$. Resultados similares a un estudio observacional de 123 pacientes ventilados realizado por Cordemans y colaboradores, ${ }^{9}$ quienes reportaron el comportamiento del cambio máximo de EVLW $\left(\Delta_{\max } E V L W\right)$, el índice de fuga capilar (CLI), la presión intraabdominal media (IAPmedia) y el manejo tardío conservador de fluidos y se demostró que un incremento persistente del índice de fuga capilar, el EVLWi y el balance hídrico están asociados a un desenlace fatal. En nuestro estudio se estableció un valor de corte de EVLWi de $11 \mathrm{~mL} /$ $\mathrm{kg}$, ya que ha sido referido como el mejor predictor de mortalidad con sensibilidad de $60 \%$, especificidad de $57 \%$ y valor predictivo positivo de $61 \% .^{8}$ De esta manera se corrobora la asociación entre el EVLWi mayor de $11 \mathrm{~mL} / \mathrm{kg}$ y la mortalidad en sepsis pulmonar y no pulmonar.

\section{CONCLUSIONES}

Es indudable el valor pronóstico del EVLWi medido por termodilución transpulmonar en pacientes sépticos así como el balance hídrico positivo; a pesar de existir escasa información, hasta el momento no se ha observado asociación de ambos parámetros, aunque no se descarta que ésta exista; sin embargo, no podemos afírmalo, ya que las múltiples intervenciones realizadas para lograr una negativización de los balances hídricos pueden influir en su medida así como la escasa disponibilidad de métodos para medir el EVLWi, razón por la cual se deben realizar más estudios para comprobar la correlación lineal o variable que existe entre estos dos importantes valores predictivos de mortalidad.

\section{BIBLIOGRAFÍA}

1. Acheampong A, Vincent JL. A positive fluid balance is an independent prognostic factor in patients with sepsis. Crit Care. 2015;19(1):251. doi: 10.1186/s13054-015-0970-1.

2. Mihm FG, Feeley TW, Jamieson SW. Thermal dye double indicator dilution measurement of lung water in man: comparison with gravimetric measurements. Thorax. 1987;42(1):72-76. doi: 10.1136/thx.42.1.72.

3. Mihm FG, Feeley TW, Rosenthal MH, Lewis F. Measurement of extravascular lung water in dogs using the thermal-green dye indicator dilution method. Anesthesiology. 1982;57(2):116-122. doi: 10.1097/00000542-198208000-00009.

4. Monnet X, Teboul JL. Transpulmonary thermodilution: advantages and limits. Crit Care. 2017;21(1):147. doi: 10.1186/ s13054-017-1739-5. 
5. Eichhorn V, Goepfert MS, Eulenburg C, Malbrain ML, Reuter DA. Comparison of values in critically ill patients for global end-diastolic volume and extravascular lung water measured by transcardiopulmonary thermodilution: a meta-analysis of the literature. Med Intensiva. 2012;3:467-474. doi: 10.1016/j. medin.2011.11.014.

6. Sakka SG, Klein M, Reinhart K, Meier-Hellmann A. Prognostic value of extravascular lung water in critically ill patients. Chest. 2002;122(6):2080-2086. doi: 10.1378/chest.122.6.2080.

7. Díaz-Rubia L, Ramos-Sáez S, Vázquez-Guillamet R, et al. Efficacy of an extravascular lung water-driven negative fluid balance protocol. Med Intensiva. 2015;39(6):345-351. doi: 10.1016/j.medin.2014.07.008.

8. von Elm E, Altman DG, Egger M, et al. The Strengthening the Reporting of Observational Studies in Epidemiology (STROBE) statement: guidelines for reporting observational studies. PLOS Med. 2007;4(10):e296.

9. Cordemans C, De Laet I, Van Regenmortel N, et al. Fluid management in critically ill patients: the role of extravascular lung water, abdominal hypertension, capillary leak, and fluid balance. Ann Intensive Care. 2012;2(Suppl 1):S1. doi: 10.1186/21105820-2-S1-S1.

Conflicto de intereses: Ninguno.

Correspondencia:

Dra. Sandybell Sosa Santos

Av. Instituto Politécnico Nacional Núm. 5160,

Col. Magdalena de las Salinas, 07760,

Alcaldía Gustavo A. Madera,

Ciudad de México, México.

Teléfono: 55-5747-7560, ext. 7456

E-mail: sandy_sosa88@hotmail.com 